\title{
A Packet Loss Compliant Logic-Based Communication Algorithm for Cooperative Path-Following Control *
}

\author{
Francisco C. Rego ${ }^{*, * *}$ A. Pedro Aguiar ${ }^{*, * *}$ António M. Pascoal ${ }^{*, * * * *}$ \\ * Laboratory of Robotics and Systems in Engineering and Science (LARSyS), \\ IST/University of Lisbon, Lisbon, Portugal. \\ ** École Polytechnique Fédérale de Lausanne (EPFL), Lausanne, \\ Switzerland. \\ ${ }^{* * *}$ Faculty of Engineering, University of Porto (FEUP), Porto, Portugal. \\ **** Adjunct Scientist, National Institute of Oceanography (NIO), Goa,
} India.

\begin{abstract}
We introduce an event driven communication logic for decentralized control of a network of robotic vehicles (agents). The strategy proposed is robust to packet losses and drives the vehicles to predefined paths while holding a desired geometric formation pattern. To this effect, the paper extends an existing cooperative path following framework to consider the practical case where communications among the vehicles occur at discrete instants, instead of continuously. The introduced communication logic takes into account the topology of the communication network, the fact that communications are discrete, and the cost of exchanging information. We also address explicitly communication losses and bounded delays. Conditions are derived under which the overall closed loop system is input-to-state practically stable. The communication logic is applied to a cooperative path-following control system of multiple underactuated autonomous marine robots. Simulation results are presented and discussed.
\end{abstract}

\section{INTRODUCTION}

Cooperative path following $(\mathrm{CPF})$ is at the root of many practical applications of multiple networked autonomous vehicles. The CPF problem consists of, given $n$ autonomous vehicles and assigning a path to each vehicle, deriving control laws to drive and maintain the vehicles in their paths at a common desired speed profile, holding a specified formation pattern. In the literature, Ghabcheloo et al. [2009], Ihle et al. [2006], Skjetne et al. [2002] offer a theoretical overview of the subject and introduce techniques to solve the CPF problem.

Different solutions to the CPF and similar problems can be found in Giulietti et al. [2000], Stilwell and Bishop [2000], Ogren et al. [2002], Jadbabaie et al. [2003], Moreau [2005], Dong [2011], and Ma and Zhang [2010]. An interesting strategy consists of decoupling the CPF problem into i) a path-following (PF) problem, where the goal is to derive closed loop control laws to drive each vehicle to its path at the reference speed profile and ii) a multiple vehicle coordination problem, where the objective is to adjust the speed of each vehicle so as to achieve the desired formation pattern. The PF problem has been widely addressed in the literature, see for example Skjetne et al. [2004], Dagci et al. [2003], Soetanto et al. [2003], Plaskonka [2012]. The coordination problem, however, requires further study to address the limitations of the communication network among vehicles. In the literature, some of these issues have been addressed using graph theory to model the communication network and Lyapunov-based techniques to cope with intermittent

* This work was supported in part by projects CONAV/FCT-PT (PTDC/EEACRO/113820/2009), FCT (PEst-OE/EEI/LA0009/2011), and MORPH (EU FP7 under grant agreement No. 288704).

The first author benefited from a PhD scholarship of the Foundation for Science and Technology (FCT), Portugal communication failures and switching topologies Ghabcheloo et al. [2006, 2009], Ihle et al. [2006], Moreau [2005].

As in Aguiar and Pascoal [2007], this paper extends the CPF framework discussed in Ghabcheloo et al. [2009] to take into account that communication between vehicles occur at discrete instants, instead of continuously. To minimize the frequency of information exchange between vehicles, we borrow from the ideas in Xu and Hespanha [2006], Yook et al. [2002] who consider distributed control systems, where the controller for each system uses the states of its own system and estimates of the states of the systems it communicates with. The communication strategy considered assumes that each system has an internal filter of its own state, synchronized with the filter of its state contained in the systems it communicates with. The communication logic consists of only transmitting information when the difference between the actual and the estimated states exceed a certain level. With this method, communication occurs asynchronously at discrete instants of time.

The main contribution of this paper is to extend the framework of Aguiar and Pascoal [2007] to consider packet losses during communication among vehicles.

Section 2 describes, albeit at an informal level, the cooperative path following problem that is at the core of the research work reported in the paper. The section borrows considerably from the results in Aguiar and Pascoal [2007]. Section 3 introduces a controller design which solves the cooperative path-following problem for a class of Autonomous Marine Vehicles (AMVs) which evolve in 2D. Finally, in section 4 we present simulation results of a formation of AMVs equipped with the controllers introduced in section 3 . The conclusions and recommendations for future work are included in section 5. 


\section{PROBLEM STATEMENT}

In this paper we introduce a new architecture for cooperative path-following control systems (CPFCS). Given $n$ agents (vehicles), the first objective of a CPFCS is to drive the output $z_{i}$ of agent $i ; i=1,2, n$ and make it converge to and remain inside a tube centered about a desired path $z_{d_{i}}\left(\gamma_{i}\right)$, parameterized by $\gamma_{i} \in \mathbb{R}$, while ensuring that its rate of progression $\dot{\gamma}_{i}$ also converges to and remain inside a tube centred about a desired speed profile $v_{r}\left(\gamma_{i}, t\right)$. In addition, a CPFCS must guarantee that the path variables $\gamma_{i} ; i=1,2, n$, are synchronized, that is, all the coordination errors $\gamma_{i}-\gamma_{j}$ converge to and remain inside a ball around the origin. The path variables $\gamma_{i}$ may also be called parametrising variables, path-following variables or, given their role in the coordination of agents, coordination states. Notice that the desired speed profiles are defined in terms of the rates of evolution of the $\gamma_{i}$ variables, not the inertial speeds and are equal for all vehicles. See Aguiar and Pascoal [2007] for an introduction to this circle of ideas and the basic notation.

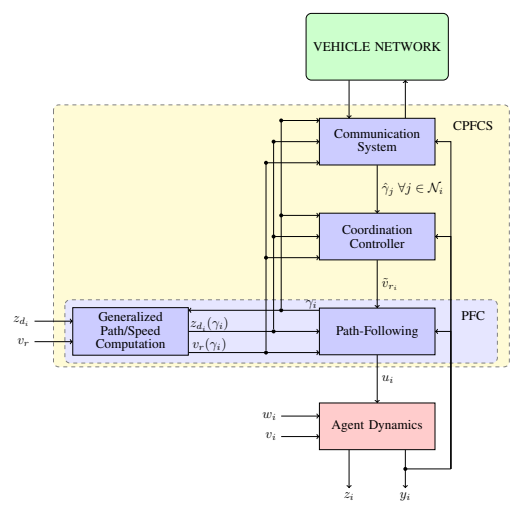

Fig. 1. Coordinated path-following control system architecture

The architecture for a general CPFCS proposed in this paper is shown in figure 1 . The CPFCS architecture consists of three interconnected subsystems:

Path-following controller a dynamical system whose inputs are a path $z_{d_{i}}$, a desired speed profile $v_{r}$ that is common to all agents, and the agent's output $y_{i}$. Its output is the agent's input, computed so as to make it follow the path at the assigned speed. A path-following controller which achieves this objective is considered to solve the path-following $(P F)$ problem. In preparation for the connection with the coordination controller, this system produces also a generalized path $z_{\boldsymbol{d}_{i}}$, which contains $z_{d_{i}}$ and its partial derivatives with respect to $\gamma_{i}$ up to a certain order, a generalized speed profile $\boldsymbol{v}_{\boldsymbol{r}_{\boldsymbol{i}}}$, which contains $v_{r}\left(\gamma_{i}\right)$ and its partial derivatives with respect to $\gamma_{i}$ also up to a certain order, and a generalized path variable $\gamma_{i}$, which contains $\gamma_{i}$ and the time derivatives of $\gamma_{i}$, also until a determined order. Furthermore, it accepts corrective speed action from the coordination controller via the signal $\tilde{v}_{r_{i}}$. Notice that the dynamics of the parametrising variable $\gamma_{i}$ are defined internally at this stage and play the role of an extra design knob to tune the performance of the path-following control law.

Coordination controller a dynamical system whose inputs are the plant output $y_{i}$, the desired generalized path $z_{\boldsymbol{d}_{i}}$ and speed profile $\boldsymbol{v}_{\boldsymbol{r}_{i}}$, the generalized path variable $\gamma_{i}$, and estimates of the coordination states $\gamma_{j} ; j \in \mathcal{N}_{i}$, where $\mathcal{N}_{i}$, denotes the set of agents that agent $i$ communicates with. Its output is the correction speed signal $\tilde{v}_{r_{i}}$, which is used to synchronize agent $i$ with its neighbours. A coordination controller which achieves this objective is considered to solve the coordination control $(C C)$ problem. If both the path-following controllers and the coordination controllers achieve their objectives simultaneously they are said to solve the coordinated path-following $(C P F)$ problem.

Logic-based communication system a logic-based dynamical system that makes the interface with the network system through which the agents output $y_{i}$, the generalized desired path $z_{d_{i}}$, the generalized speed profile $\boldsymbol{v}_{\boldsymbol{r}_{i}}$, and the generalized path variable $\gamma_{i}$ can be communicated to the neighbour agents. Its output is an estimate of the general coordination states of the neighbouring agents $\hat{\gamma}_{j}^{i}, j \in \mathcal{N}_{i}$. A communication system which achieves the objective of keeping the estimation errors $\tilde{\gamma}_{j}^{i}:=\hat{\gamma}_{j}^{i}-\gamma_{j}$ uniformly bounded is considered to solve the communication problem.

Inspired by the communication logic proposed in $\mathrm{Xu}$ and Hespanha [2006], each communication subsystem is composed by a bank of estimators and a communication logic. The estimators run in open-loop most of the time but are sometimes reset (not necessarily periodically) to correct their state when measurements are received through the network. The communication logic is responsible for determining for each agent, using an internal estimator, how well the other agents from the communication topology can predict the value of its local coordination state and decide when it should communicate the actual measured value to its neighbours. As in Xu and Hespanha [2006], the banks of estimators running in the different agents are synchronized, that is, the state estimate of each agent is the same as that of the corresponding neighbours.

A formal definition of the PF, CC, CPF and communication problems can be found in Aguiar and Pascoal [2007].

\section{CONTROLLER DESIGN}

In what follows, without loss of generality, we consider the case where the vehices evolve in 2D.

\subsection{Path-following controller}

The path-following controller considered in this paper is extensively described in Vanni et al. [2008]. Let $\boldsymbol{p}_{d}$ be the position of the target, and $v_{r}$ its desired rate of progression. We decompose the motion-control problem into an inner-loop dynamic task, which consists of making the vehicle's surge speed $u$ and heading angle $\psi$ track desired references $u_{d}$ and $\psi_{d}$ respectively, and an outer-loop kinematic task on the desired speed reference $\boldsymbol{u}_{d}:=\left[u_{d} \dot{\psi}_{d}\right]^{\prime}$, which $i$ ) regulates the evolution of the virtual target and ii) assigns the reference speed so as to achieve convergence to the path.

Before proceeding further we have to define the reference frames used in this paper. The body-fixed reference frame $\{\mathcal{B}\}$ is a reference frames centered at the vehicle with the $x$ axis pointing towards the vehicle heading, that is, the rotation angle between the inertial coordinates and the body-fixed reference frame is the heading angle $(\psi)$. The reference heading coordinates $\left\{\mathcal{B}_{d}\right\}$ is a reference frames centered at the vehicle with the $x$ axis pointing towards the reference heading, that is, the rotation angle between the inertial coordinates and the reference heading coordinates is the reference heading angle $\left(\psi_{d}\right)$. 
We can write the kinematic equations of motion of a vehicle moving in the horizontal plane by using a global inertial coordinate frame $\{\mathcal{U}\}$ and a body-fixed coordinate frame $\{\mathcal{B}\}$, with origin at the vehicle's center of mass, resulting in

$$
\dot{\boldsymbol{p}}=R(\psi) \boldsymbol{v}
$$

where $\boldsymbol{p}$ and $\boldsymbol{v}$ are defined as $\boldsymbol{p}:=\left[\begin{array}{ll}x & y\end{array}\right]^{\prime}$ and $\boldsymbol{v}:=\left[\begin{array}{ll}u & v\end{array}\right]^{\prime}$, where $u$ and $v$ are body-fixed frame components of the vehicle's velocity, $x$ and $y$ are the inertial cartesian coordinates of its center of mass, $\psi$ defines its orientation (heading angle) and $R(\psi)$ is the orthonormal transformation matrix from $\{\mathcal{B}\}$ to $\{\mathcal{U}\}$. In the presence of a constant ocean current, $\boldsymbol{v}$ is the sum of the vehicle's velocity with respect to the water $\boldsymbol{v}_{w}:=\left[\begin{array}{ll}u_{w} & v_{w}\end{array}\right]^{\prime}$ and the water current velocity $\boldsymbol{v}_{c}:=\left[\begin{array}{ll}u_{c} & v_{c}\end{array}\right]^{\prime}$, both expressed in the body-fixed reference frame.

In what follows we assume that the inner-loop controller satisfies the following stability property:

Property 1. Let $\tilde{u}:=u-u_{d}$ and $\tilde{\psi}:=\psi-\psi_{d}$ be the speed errors. Then $\tilde{u}$ and $\tilde{\psi}$ are globally uniformly ultimately bounded (GUUB).

We also assume that each vehicle contains an observer for the lateral water current velocity $v_{c}$ which satisfies the following property:

Property 2. Let $\tilde{v}_{c}:=v_{c}-\hat{v}_{c}$ be the estimation error of the lateral water current velocity estimator. Then $\tilde{v}_{c}$ is GUUB.

In addition, we introduce the following constraint.

Property 3. The measurements of the vehicle's velocity with respect to the water $\boldsymbol{v}_{w}$ are not available.

Before writing the path-following control law we define the position error $e$ as the difference between the positions of the vehicle and of the virtual target expressed in the reference heading coordinates $\left\{\mathcal{B}_{d}\right\}$, that is $\boldsymbol{e}:=R_{d}^{\prime}\left(\boldsymbol{p}(t)-\boldsymbol{p}_{d}(\gamma(t))\right)$, where $R_{d}$ is the orthonormal transformation matrix from $\left\{\mathcal{B}_{d}\right\}$ to $\{\mathcal{U}\}$.

We also need to define the virtual target speed error as $e_{\dot{\gamma}}:=$ $\dot{\gamma}-v_{r}(\gamma)$.

The following path-following strategy borrows from the technique of backstepping.

Lemma 1. Consider the vehicle model described by (1), with finite values of $\sup _{\gamma \in \mathbb{R}}\left|\frac{\partial \boldsymbol{p}_{d}(\gamma)}{\partial \gamma}\right|$ and $\sup _{\gamma \in \mathbb{R}}\left|\frac{\partial \boldsymbol{p}_{d}(\gamma)}{\partial \gamma} v_{r}(\gamma)\right|$, in closed-loop with the output feedback control law composed by an inner loop that satisfies property 1 , a lateral current estimator which satisfies property 2 , and the outer loop given by

$$
\ddot{\gamma}=-k_{\omega}\left(\dot{\gamma}-v_{r}(\gamma)-\tilde{v}_{r}\right)+\dot{v}_{r}(\gamma)+\frac{1}{c_{\omega}}(\boldsymbol{e}-\boldsymbol{\delta})^{\prime} R_{d}^{\prime} \frac{\partial \boldsymbol{p}(\gamma)}{\partial \gamma}
$$

where we introduce a constant design vector $\delta:=[\delta, 0]^{\prime}, \delta<0$ and

$$
\boldsymbol{u}_{d}=\Delta^{-1}\left(-K_{k}(\boldsymbol{e}-\boldsymbol{\delta})-\left[\begin{array}{c}
0 \\
\hat{v}_{c}
\end{array}\right]+R_{d}^{\prime} \frac{\partial \boldsymbol{p}_{d}(\gamma)}{\partial \gamma}\left(v_{r}(\gamma)+\tilde{v}_{r}\right)\right)
$$

where $K_{k}:=\operatorname{diag}\left\{k_{x}, k_{y}\right\}, c_{\omega}>0$ and $\Delta:=\left[\begin{array}{cc}1 & 0 \\ 0 & -\delta\end{array}\right]$.

Then, for appropriate choices of $k_{x}, k_{y}$ and $k_{\omega}$, the control laws (1-2) solve robustly the path-following problem, that is, the closed-loop state is input-to-state practically stable (ISpS) with respect to input $\tilde{v}_{r}$.

With this strategy the evolution of the position of the virtual target $\boldsymbol{p}_{d}$ depends on the position error $(\boldsymbol{e}-\boldsymbol{\delta})$. As a consequence, if the vehicle is ahead/behind the desired position the virtual target moves faster/slower.

\subsection{Coordination controller design}

From the theory of consensus of distributed systems, a natural choice for the coordination control law would be $\tilde{v}_{r_{d_{i}}}=$ $-k \sum_{j \in \mathcal{N}_{i}}\left(\gamma_{i}-\gamma_{j}\right)$, where $k$ is a positive scalar (the so-called neighbouring rule). To reduce the communication rate using a logic based dynamical system, we will lift the assumption that each agent receives information from its neighbourhoods continuously. We assume instead that it relies on estimates. Therefore, the coordination control law becomes

$$
\tilde{v}_{r_{i}}=-k \sum_{j \in \mathcal{N}_{i}}\left(\gamma_{i}-\hat{\gamma}_{j}^{i j}\right)
$$

where $\hat{\gamma}_{l}^{i j}$ is an estimate of $\gamma_{l}$ running on agent $i$ synchronized with agent $j$.

We are now ready to state the main result of this section.

Lemma 2. Consider the closed-loop system $\Sigma_{C L}$ composed by $n$ agents of the form (1) with inner loops satisfying property 1 , a lateral current estimator which satisfies property 2 , and the path-following controller and coordination controller defined by (1)-(2) and (3) respectively. If the communication graph is connected then, for appropriate selections of $k_{k}, k_{x}, k_{y}, c_{\omega}$ and $k, \Sigma_{C L}$ solve robustly the CPF problem.

\subsection{Logic-based communication system}

1) No delayed information: Let $t_{k}^{[i j]}, k>0$ denote the instants of time at which agent $i$ transmits data to $j$ or $j$ transmits data to $i, \mathcal{L}_{i j}$ the agents with the coordination state estimated in agent $i$ synchronized with agent $j$, and $\beta_{k}^{i j} \in\{i, j\}$ the agent which sent data at $t_{k}^{[i j]}$. Following the procedure described in Section 2 and taking into account the dynamic equations of the coordination subsystem, we propose for each agent $i$ the following logic-based communication system:

-For $t_{k}^{[i j]} \leq t<t_{k+1}^{[i j]}$

$$
\begin{gathered}
\dot{\hat{\gamma}}_{l}^{i j}=v_{r}\left(\hat{\gamma}_{l}^{i j}\right)+\hat{\tilde{v}}_{r_{l}}^{i j}, \quad l \in \mathcal{L}_{i j} \\
\hat{\tilde{v}}_{r_{l}}^{i j}=-k_{l} \sum_{m \in \mathcal{N}_{l} \cap \mathcal{L}_{i j}}\left(\hat{\gamma}_{l}^{i j}-\hat{\gamma}_{m}^{i j}\right), \quad l \in \mathcal{L}_{i j}
\end{gathered}
$$


-For $t=t_{k}^{[i j]}$

$$
\hat{\gamma}_{l}^{i j}\left(t_{k}^{[i j]+}\right)=\hat{\gamma}_{l}^{\beta_{k}^{i j}}
$$

where, for a signal $x(t)$, we define $x\left(t^{+}\right):=\lim _{s \rightarrow t^{+}} x(s)$ and $\hat{\gamma}_{j}^{i}$ is defined as

$$
\hat{\gamma}_{j}^{i}:= \begin{cases}\gamma_{i} & \text { if } j=i \\ \hat{\gamma}_{j}^{i \alpha_{i j}} & \text { otherwise }\end{cases}
$$

with $\alpha_{i j} \in \mathcal{N}_{i}$ and $\alpha_{i j}=j$ if $j \in \mathcal{N}_{i}$. We will consider $\mathcal{L}_{i j}$ containing all the agents in the formation. However, other choices such as $\mathcal{L}_{i j}=\{i, j\}$ could also be considered. In which case we would have $\hat{\tilde{v}}_{r_{i}}^{i j}=-k\left(\hat{\gamma}_{i}^{i j}-\hat{\gamma}_{j}^{i j}\right)$ and $\hat{\tilde{v}}_{r_{j}}^{i j}=-k\left(\hat{\gamma}_{j}^{i j}-\right.$ $\left.\hat{\gamma}_{i}^{i j}\right)$.

To solve robustly the communication problem we introduce the communication threshold $\mu>0$ and $\tilde{\gamma}_{i}^{j}:=\hat{\gamma}_{i}^{j}-\gamma_{i}$ and use the following logic: agent $i$ transmits to $j$ a message composed by $\left\{\hat{\gamma}_{l}^{i}: l \in \mathcal{L}_{i j}\right\}$ at time $t_{k}^{[i j]}$ when $\tilde{\gamma}_{i}^{j}\left(t_{k}^{[i j]}\right) \geq \mu$. Since the message was sent by agent $i$ we define the index $\beta_{k}^{i j}=i$, otherwise it would be $\beta_{k}^{i j}=j$.

Notice however that we did not consider the case where $\tilde{\gamma}_{i}^{j}\left(t_{k}^{[i j]}\right) \geq \mu$ and $\tilde{\gamma}_{j}^{i}\left(t_{k}^{[i j]}\right) \geq \mu$, that is, when both agents send messages at the same time. To handle this case we consider that each communication link has a primary and a secondary agent. Considering without loss of generality that $i$ is a primary agent on link $i j$, then $\hat{\gamma}_{l}^{i j}$ and $\hat{\gamma}_{l}^{j i}, l \in \mathcal{L}_{i j}$ are updated as

$$
\hat{\gamma}_{l}^{i j}\left(t_{k}^{[i j]+}\right)=\hat{\gamma}_{l}^{j i}\left(t_{k}^{[i j]+}\right)= \begin{cases}\hat{\gamma}_{l}^{j} & \text { if } \alpha_{j l} \neq i \\ \hat{\gamma}_{l}^{i} \text { if } \alpha_{j l}=i\end{cases}
$$

With this method the post reset values of $\tilde{\gamma}_{i}^{j}$ and $\tilde{\gamma}_{j}^{i}$ are equal to zero and therefore are also bounded in this case.

2) Delayed information: We now consider the case where the communication channels have bounded, time-varying and nonhomogeneous delays. Consider the following situation: agent $i$ sends data to $j$ at time $t_{k}^{[i j]}$, and agent $j$ receives it at time $t_{k}^{[i i]}+\tau_{k}^{i j}$. We assume that $\tau_{k}^{i j} \leq \bar{\tau}$ for any admissible $i j$ and $k$, where $\bar{\tau}>0$ is known a priori.

Suppose that at time $t_{k}^{[i j]}$ agent $i$ transmits to agent $j$ a message, which contains contains $t_{k}^{[i j]}$ and $\left\{\hat{\gamma}_{l}^{i}: l \in \mathcal{L}_{i j}\right\}$. Then, the internal filters states $\left\{\hat{\gamma}_{l}^{i}: l \in \mathcal{L}_{i j}\right\}$ cannot be immediately updated. This is because we must guarantee that the value of the state estimate $\hat{\gamma}_{l}^{i j}$ will always remain equal to the corresponding state estimate running in agent $j, \hat{\gamma}_{l}^{j i}$. To this effect, both estimates can only be updated $\tau:=\bar{\tau}+\tau_{\text {tol }}$ time units after the message was sent, for some $\tau_{\text {tol }}>0$. Upon receiving $t_{k}^{[i j]}$, the coordination state estimate $\hat{\gamma}_{l}^{[i j]}$ running in agent $j$ should be updated at time $t=t_{k}^{[i j]}+\tau$ to

$$
\hat{\gamma}_{l}^{i j}\left(t_{k}^{[i j]}+\tau\right)=\hat{\gamma}_{l}^{i}\left(t_{k}^{[i j]}\right)+\tau v_{r}\left(\hat{\gamma}_{l}^{i}\left(t_{k}^{[i j]}\right)\right)
$$

With the above procedure, we guarantee that the estimators are always synchronized.
We still need to guarantee that $\tau$ is sufficiently small and the communication threshold $\mu$ is selected to be sufficiently large so as to guarantee that the post-reset value of $\tilde{\gamma}_{i}^{j}$ satisfies $\tilde{\gamma}_{i}^{j}<$ $\mu$.

Although in Aguiar and Pascoal [2007] a more elaborate update scheme was used, which considers variations in $v_{r}$ and the control effort, in this paper we consider equation (9) for ease of implementation. Note that the results still hold if we guarantee that $\tau$ is sufficiently small.

We also have to consider the case where, on link $i j$ an agent tries to send a message while the other agent has already sent one message during the $\tau$ previous time units. For each link we consider a primary and a secondary agent, as was done before. Considering without loss of generality that $i$ is a primary agent on link $i j$, then the message sent by agent $j$ is ignored and if $\tilde{\gamma}_{j}^{i}\left(t_{k}^{[i j]}+\tau\right) \geq \mu$ holds, then another message is sent by agent $j$ at time $t_{k+1}^{[i j]}=t_{k}^{[i j]}+\tau$.

The estimation error $\tilde{\gamma}_{j}^{i}\left(t_{k+1}^{[i j]}+\tau\right)$ is bounded assuming that the time delay is bounded. We can guarantee that this technique is valid if $\tau$ is sufficiently small and the communication threshold $\mu$ is selected to be sufficiently large so as to guarantee that $\tilde{\gamma}_{j}^{i}$ satisfies $\tilde{\gamma}_{j}^{i}\left(t_{k}^{[i j]}+\tau\right)<\mu$.

\section{3) Communication losses:}

To make the communication system robust to limited communication losses we require each agent to send a reply upon receiving a message. The agent which sent the message only updates its estimators if the reply is received $\tau:=2 \bar{\tau}+\tau_{\text {tol }}$ time units after the message was sent, for some $\tau_{\text {tol }}>0$, otherwise another message is sent.

Consider the case where at time $t_{k}^{[i j]}$ agent $i$ transmits to agent $j$ a message, which contains $t_{k}^{[i j]}$ and $\left\{\hat{\gamma}_{l}^{i}: l \in \mathcal{L}_{i j}\right\}$. Upon receiving the message and sending a reply, the coordination state estimates $\hat{\gamma}_{l}^{j i}$ running in agent $j$ should be updated at time $t=t_{k}^{[i j]}+\tau$ to

$$
\hat{\gamma}_{l}^{j i}\left(t_{k}^{[i j]}+\tau\right)=\hat{\gamma}_{l}^{i}\left(t_{k}^{[i j]}\right)+\tau v_{r}\left(\hat{\gamma}_{l}^{i}\left(t_{k}^{[i j]}\right)\right)
$$

If the reply was received by agent $i$ then the coordination state estimates $\hat{\gamma}_{l}^{i j}$ running in agent $i$ should be also update at the same time as $\hat{\gamma}_{l}^{i j}\left(t_{k}^{[i j]}+\tau\right)=\hat{\gamma}_{l}^{j i}\left(t_{k}^{[i j]}+\tau\right)$, otherwise, a reply is sent at $t_{k+1}^{[i j]}=t_{k}^{[i j]}+\tau$. Note that if agent $i$ did not receive the reply, then there is a brief period when the estimators are desynchronized, that is $\hat{\gamma}_{l}^{i j} \neq \hat{\gamma}_{l}^{j i}$, however the equality is replaced after the reply of the next message is received.

We must now consider the case of conflicting messages. If on link $i j$ an agent tries to send a message while the other agent has already sent one message during the $\tau$ previous time units, we consider, for each link, a primary and a secondary agent. Considering, without loss of generality, that $i$ is a primary agent on link $i j$, then the message sent by agent $j$ is ignored and, if $\left|\tilde{\gamma}_{j}^{i}\left(t_{k}^{[i j]}+\tau\right)\right| \geq \mu$ holds, then another message is sent by agent $j$ at time $t_{k+1}^{[i j]}=t_{k}^{[i j]}+\tau$.

If we can guarantee that for two consecutive messages sent one reply is received then, if $\bar{\tau}$ and $\tau_{\text {tol }}$ are sufficiently small and the 
communication threshold $\mu$ is selected to be sufficiently large so as to guarantee that $\tilde{\gamma}_{i}^{j}$ satisfies $\left|\tilde{\gamma}_{i}^{j}\left(t_{k}^{[i j]}+\tau\right)\right|<\mu$, then the estimation error is bounded.

The communication logic for the case with delayed information and communication losses is illustrated in Figure 2.

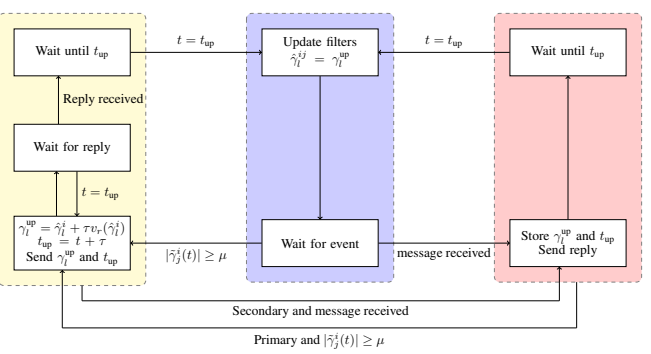

Fig. 2. Communication logic diagram.

We can now state the main result of this paper. We omit the proof.

Theorem 1. Consider the overall closed-loop system $\Sigma_{C L}$ composed by $n$ agents of the form (1), with inner loop controllers satisfying property 1 , a lateral current estimator which satisfies property 2, the proposed CPF controller under the assumptions of lemma 2, and logic-based communication system, in the presence of delayed information and communication losses. Assume there exists a number $m \in \mathbb{N}$ for which it can be guaranteed that if the number of consecutive messages sent by an agent is greater than or equal to $m$, at least one reply to one of those messages is received. Then, for appropriately chosen path-following control gains and coordination control gains and for sufficiently small time delays and communication threshold $\mu$, the overall closed loop system solves the CPF problem.

\section{SIMULATION}

To simulate the performance of the designed CPFCS we used a Simulink model of an Autonomous Marine Vehicle of the MEDUSA_S class, built at IST, with the inner loop controller for heading and speed described in Ribeiro [2011].

We consider a lateral water current observer with the law $\dot{\hat{v}}_{c}=$ $k_{v_{c}}\left\{\left[\begin{array}{ll}0 & 1\end{array}\right] R_{d}^{\prime} \dot{\boldsymbol{p}}-\hat{v}_{c}\right\}$.

The formation considered here is composed by three agents where agent 1 communicates with 2 , agent 2 communicates with agents 1 and 3 and therefore agent 3 only communicates with agent 2 . Agent 2 is a primary agent on both links, 12 and 23. In this case there is no freedom on the selection of $\alpha_{i j}$. We have in agent $1 \hat{\gamma}_{2}^{1}:=\hat{\gamma}_{2}^{12}, \hat{\gamma}_{3}^{1}:=\hat{\gamma}_{3}^{12}$, in agent $2 \hat{\gamma}_{1}^{2}:=\hat{\gamma}_{1}^{21}$, $\hat{\gamma}_{3}^{2}:=\hat{\gamma}_{3}^{23}$, and in agent $3 \hat{\gamma}_{1}^{3}:=\hat{\gamma}_{1}^{32}, \hat{\gamma}_{2}^{3}:=\hat{\gamma}_{2}^{32}$. The three vehicles are required to maintain a triangular formation with a $15 \mathrm{~m}$ side. The formation will follows straight trajectories with a U-turn upon reaching $x=80 \mathrm{~m}$ (In order to access the full potential of this control architecture a temporary engine failure of agent 1 is simulated at $t=500 \mathrm{~s}$ with a recovery at $t=600 \mathrm{~s})$. In this simulation, no currents were considered.

The simulated paths of the three vehicles can be seen in Figure 3

The communication instants during engine failure can be seen in Figure 4. In Figures 4 the value 1 represents a sent message and -1 represents a reply to a received message.

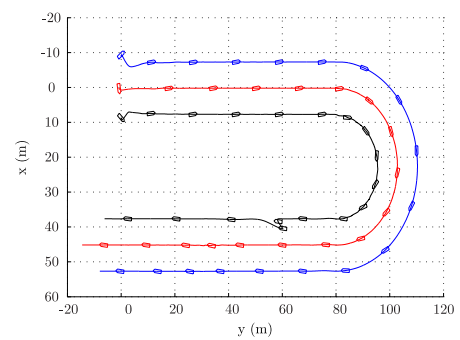

Fig. 3. Vehicle paths.

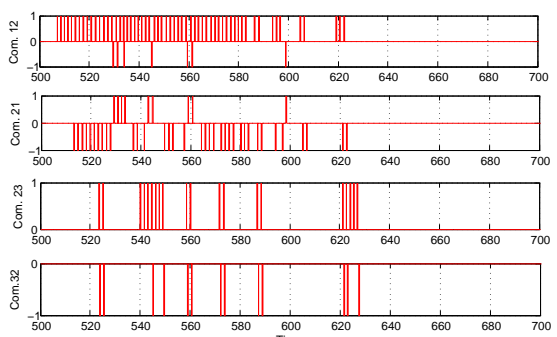

Fig. 4. Communication instants.

It can be observed that agent 1 communicates heavily with agent 2 , agent 2 communicates moderately with agents 1 and 3 , and agent 3 only receives messages from agent 2 . The reason behind these rates of communication between vehicles will be explained next.

The estimated coordination states computed by each communication logic block during engine failure are shown in Figures $5-8$.

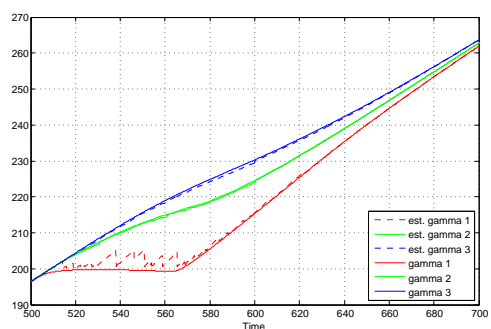

Fig. 5. Communication logic output $\hat{\gamma}_{i}^{12}$.

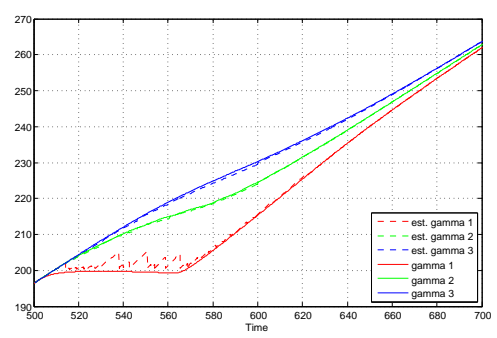

Fig. 6. Communication logic output $\hat{\gamma}_{i}^{21}$.

Since the gamma kinematics is designed to reduce the pathfollowing errors, that is, to keep the desired position close to the real agent position, $\gamma_{1}$ goes to a stop a few seconds after engine failure. Since the expected gamma kinematics of the filters in the communication logic block do not account for the path-following error, the estimation errors of $\gamma_{1}$ become greater than in normal conditions, explaining the heavy need for communications. The gamma kinematics of agent 2 impose a strong deceleration due to the effect of agent 1 , therefore there is a slight oscillatory behaviour which degrades the filter 


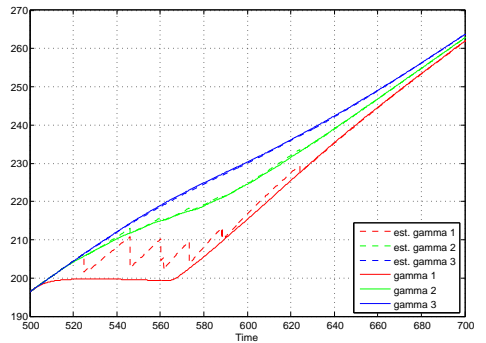

Fig. 7. Communication logic output $\hat{\gamma}_{i}^{23}$.

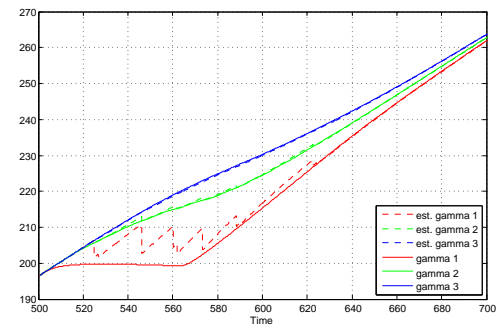

Fig. 8. Communication logic output $\hat{\gamma}_{i}^{32}$.

performance, and therefore there is a small need of communication. Agent 3 is only affected by agent 2 and therefore the decelerations imposed by the gamma kinematics are relatively weak, then the path-following has no difficulty in follow $p_{d}(\gamma)$ and therefore there is no need for updating the filters for $\gamma_{3}$. From Figures 3, and 5-8 it can be observed that coordination is achieved while each agent follows its assigned path.

\section{CONCLUSION}

A distributed control system for multiple vehicles was introduced, which makes use of logic-based communications with low bandwidth and packet loss robustness requirements to solve robustly the CPF problem, i.e. to steer and maintain the vehicles at defined paths at a common desired speed profile, while holding a formation pattern. The control system takes into account the topology of the communication network between vehicles and the fact that communication takes place at discrete instants of time, instead of continuously. The communication logic also considers packet losses and delays in the communication network. The methodology developed holds considerable potential for practical applications. Future work will include the execution of field tests with a small fleet of MEDUSA marine robots, that are property of IST.

\section{REFERENCES}

A.P. Aguiar and A.M. Pascoal. Coordinated path-following control for nonlinear systems with logic-based communication. In Decision and Control, 2007 46th IEEE Conference on, pages 1473-1479, 2007.

O.H. Dagci, U.Y. Ogras, and U. Ozguner. Path following controller design using sliding mode control theory. In American Control Conference, 2003. Proceedings of the 2003, volume 1, pages 903-908, june 2003.

W. Dong. On consensus algorithms of multiple uncertain mechanical systems with a reference trajectory. Automatica, 47(9):2023-2028, 2011.

R. Ghabcheloo, A.P. Aguiar, A. Pascoal, C. Silvestre, I. Kaminer, and J. Hespanha. Coordinated path-following control of multiple underactuated autonomous vehicles in the presence of communication failures. In Decision and Control, 2006 45th IEEE Conference on, pages 4345-4350, dec. 2006.

R. Ghabcheloo, A. P. Aguiar, A. Pascoal, C. Silvestre, I. Kaminer, and J. Hespanha. Coordinated path-following in the presence of communication losses and time delays. SIAM J. Control Optim., 48(1):234-265, February 2009.

F. Giulietti, L. Pollini, and M. Innocenti. Autonomous formation flight. Control Systems, IEEE, 20(6):34-44, dec 2000.

Ivar-André F. Ihle, Murat Arcak, and Thor I. Fossen. Passivitybased designs for synchronized path-following. Automatica, 43:1508-1518, 2006.

A. Jadbabaie, Jie Lin, and A.S. Morse. Coordination of groups of mobile autonomous agents using nearest neighbor rules. Automatic Control, IEEE Transactions on, 48(6):988-1001, june 2003.

C.Q. Ma and J.F. Zhang. Necessary and sufficient conditions for consensusability of linear multi-agent systems. Automatic Control, IEEE Transactions on, 55(5):1263-1268, 2010.

L. Moreau. Stability of multiagent systems with timedependent communication links. Automatic Control, IEEE Transactions on, 50(2):169-182, feb. 2005.

P. Ogren, M. Egerstedt, and Xiaoming Hu. A control lyapunov function approach to multiagent coordination. Robotics and Automation, IEEE Transactions on, 18(5):847-851, oct 2002.

Joanna Plaskonka. The path following control of a unicycle based on the chained form of a kinematic model derived with respect to the serret-frenet frame. In Methods and Models in Automation and Robotics (MMAR), 2012 17th International Conference on, pages 617-620, aug. 2012.

Jorge Ribeiro. Motion control of single and multiple autonomous martine vehicles. Master's thesis, Instituto Superior Tcnico, 2011.

R. Skjetne, S. Moi, and T.I. Fossen. Nonlinear formation control of marine craft. In Decision and Control, 2002, Proceedings of the 41st IEEE Conference on, volume 2, pages 1699-1704 vol.2, 2002.

Roger Skjetne, Thor I. Fossen, and Petar V. Kokotović. Robust output maneuvering for a class of nonlinear systems. Automatica, 40(3):373-383, 2004.

D. Soetanto, L. Lapierre, and A. Pascoal. Adaptive, nonsingular path-following control of dynamic wheeled robots. In Decision and Control, 2003. Proceedings. 42nd IEEE Conference on, volume 2, pages 1765-1770, dec. 2003.

D.J. Stilwell and B.E. Bishop. Platoons of underwater vehicles. Control Systems, IEEE, 20(6):45-52, dec. 2000.

Francesco Vanni, A Pedro Aguiar, and António M Pascoal. Cooperative path-following of underactuated autonomous marine vehicles with logic-based communication. In Proc. of NGCUV08-IFAC Workshop on Navigation, Guidance and Control of Underwater Vehicles, pages 1-6, 2008.

Yonggang $\mathrm{Xu}$ and João P. Hespanha. Communication logic design and analysis for networked control systems. In Laura Menini, Luca Zaccarian, and Chaouki T. Abdallah, editors, Current Trends in Nonlinear Systems and Control, Systems \& Control: Foundations \& Applications, pages 495-514. Birkhäuser Boston, 2006.

J.K. Yook, D.M. Tilbury, and N.R. Soparkar. Trading computation for bandwidth: reducing communication in distributed control systems using state estimators. Control Systems Technology, IEEE Transactions on, 10(4):503-518, jul 2002. 